\title{
Real-time model-based image reconstruction with a prior calculated database for electrical capacitance tomography
}

DOI:

10.1088/1361-6501/aa6221

\section{Document Version}

Accepted author manuscript

Link to publication record in Manchester Research Explorer

\section{Citation for published version (APA):}

Rodriguez Frias, M. A., \& Yang, W. (2017). Real-time model-based image reconstruction with a prior calculated database for electrical capacitance tomography. Measurement Science and Technology, 28(5), [054006]. https://doi.org/10.1088/1361-6501/aa6221

\section{Published in:}

Measurement Science and Technology

\section{Citing this paper}

Please note that where the full-text provided on Manchester Research Explorer is the Author Accepted Manuscript or Proof version this may differ from the final Published version. If citing, it is advised that you check and use the publisher's definitive version.

\section{General rights}

Copyright and moral rights for the publications made accessible in the Research Explorer are retained by the authors and/or other copyright owners and it is a condition of accessing publications that users recognise and abide by the legal requirements associated with these rights.

\section{Takedown policy}

If you believe that this document breaches copyright please refer to the University of Manchester's Takedown Procedures [http://man.ac.uk/04Y6Bo] or contact uml.scholarlycommunications@manchester.ac.uk providing relevant details, so we can investigate your claim.

\section{OPEN ACCESS}




\title{
Real-time Model-based Image Reconstruction with a Prior Calculated Database for Electrical Capacitance Tomography
}

\author{
Marco A. Rodriguez Frias and Wuqiang Yang, Fellow, IEEE \\ School of Electrical and Electronic Engineering, The University of Manchester, Manchester M13 9PL, UK \\ marco.rodriguezfrias@manchester.ac.uk; wuqiang.yang@manchester.ac.uk
}

\begin{abstract}
Image reconstruction for electrical capacitance tomography (ECT) is a challenging task due to the severely underdetermined nature of the inverse problem. A model-based algorithm tackles this problem by reducing the number of unknowns to be calculated from the limited number of independent measurements. The conventional model-based algorithm is implemented with a finite element method (FEM) to solve the forward problem at each iteration and can produce good results. However, it is time-consuming and hence the algorithm can be used for off-line image reconstruction only. In this paper, a solution to this limitation is proposed. The model-based algorithm is implemented with a database containing a set of prior solved forward problems. In this way, the time required to perform image reconstruction is drastically reduced without sacrificing accuracy, and real-time image reconstruction achieved with up to 100 frames/second. Further enhancement in speed may be accomplished by implementing the reconstruction algorithm in a parallel processing general purpose graphics process unit (GPU).
\end{abstract}

Keywords: Electrical capacitance tomography (ECT), forward problem, image reconstruction, multi-phase flow measurement.

\section{Introduction}

Electrical capacitance tomography (ECT) is characterised by being a safe, due to the lack of radiation sources, fast and inexpensive technique to image permittivity distributions. One of the major advantages of ECT is its high temporal resolution, usually 100 frames/second. Accordingly, an algorithm in real time and producing a quantitative estimation of the permittivity distribution is required.

Image reconstruction for quantification of permittivity distribution in ECT is usually performed by the utilisation of either single step algorithms like linear back-projection (LBP) or iterative algorithms like Landweber [1]. The selection of an appropriate algorithm always represents a trade-off. On one hand, single step algorithms have the advantage of producing fast results, whereas iterative algorithms require 
longer time and usually cannot be used for real-time measurement. On the other hand, images obtained by single step algorithms usually provide a qualitative description of the distribution only, whereas images by iterative algorithms can provide a quantitative description of the distribution.

Efforts have been made to improve the quality of the images obtained by implementing a variety of image reconstruction algorithms. Soleimani and Lionheart [2] reported the use of a non-linear image reconstruction algorithm. In this work, sensitivity maps used for solving the inverse problem are updated with the use of a finite element method (FEM) solver at each iteration. Ortiz-Aleman et al. [3] reported the use of simulated annealing and genetic optimisation algorithms to minimise an objective function. In this approach, the image is modified at every iteration and the forward problem is calculated using the current distribution. The result is then compared with the measurement vector. This procedure is repeated until a minimum error is reached. The forward problem is solved at each iteration using a finite volume method. Li and Yang [4] reported a method by updating the sensitivity maps, which are calculated using a finite difference method, at every iteration of the Landweber algorithm. The modelbased algorithm was proposed by Isaksen and Nordtvedt [5], with a selection of parameters capable of describing as much flow regimes as possible and hence reducing the number of unknowns to be determined by the limited measurement data. An initial guess is updated at each iteration by an optimisation routine. The forward problem is solved at every iteration by the use of a FEM solver. This process continues until the desired minimum objective function is reached. The above algorithms can generate high-quality images, allowing to estimate the permittivity distribution accurately. Nonetheless, the need to calculate either the sensitivity maps or the forward problem at every iteration represents a major drawback of time-consuming. In consequence, these algorithms are limited to be used only for off-line monitoring or for research purposes.

Klug and Mayinger [6] reported the use of a reference matrix, i.e. look-up table, to perform image reconstruction. Such a matrix contains calibration references compared to the measurement vector to identify the flow pattern and void fraction in a gas/oil flow. In such implementation, the reference matrix contains capacitance measurements for stratified, annular and central core flow patterns. The generation of a look-up table containing measurement vectors for all the possible configurations of uncontrolled flow patterns is highly impractical. First, because uncontrolled flow patterns can vary widely over a broad range of distributions. Second, a look-up table big enough to contain sets of measurements for all those possible variations would result in a vast array and the time to find a match to the measurement vector would render this approach impractical for real-time applications.

In this paper, a model-based algorithm capable of real-time and accurate estimation of parameters for the defined model is presented which is implemented by databases [7]. The previous knowledge of the 
permittivity distributions or the ability to constrain them allows the use of databases, which contain a set of prior solved forward problems, covering the full range of possible values of the parameters. The use of the databases eliminates the need to solve the forward problem at each iteration as the conventional model-based algorithm, and hence speeds up considerably the estimation of the parameters defining the permittivity distribution.

\section{Image reconstruction algorithms}

The purpose of solving the inverse problem in ECT is to determine the permittivity distribution according to a set of capacitance measurements. This task is usually performed by the use of a sensitivity matrix in linear image reconstruction algorithms. Such a sensitivity matrix represents the linearized response of the sensor to the presence of a perturbation in a homogeneous permittivity distribution [8].

LBP is a fast and simple single-step algorithm. It uses sensitivity maps to estimate the permittivity distribution according to a capacitance measurement vector. It is implemented as

$$
\hat{\mathrm{G}}=S^{T} \cdot C
$$

where $\hat{\mathrm{G}}$ is the approximate permittivity distribution and $\mathrm{S}^{\mathrm{T}}$ is the transpose of the sensitivity matrix.

In a model-based algorithm, a set of parameters is selected to define the permittivity distribution, relating the relevant characteristics of the permittivity distribution to the capacitance measurement vector. Selection of parameters is defined by the access to previous information regarding the permittivity distribution. If enough information is available, i.e. if the permittivity distribution is known or can be constrained, it is possible to use explicit models as shown in Figure 1. It is possible to implement the model-based algorithm with databases only for models with explicit parameters.

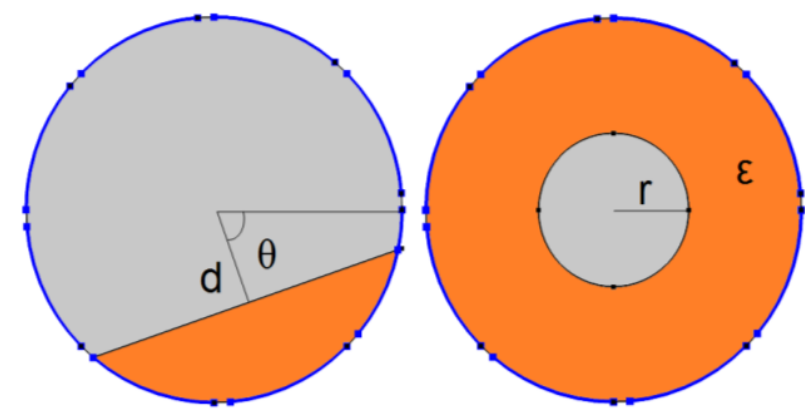

(a)

(b)

Figure 1 Explicit model parameters. Stratified distribution (a), annular distribution (b). 


\section{Databases}

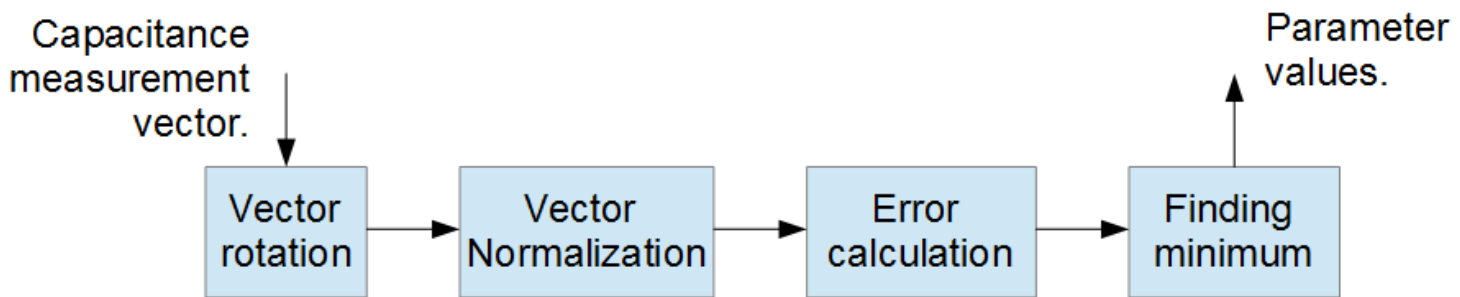

Figure 2 Model-based algorithm with databases flowchart.

\subsection{Forward Problem}

The purpose of solving the forward problem in ECT is to calculate the inter-electrode capacitance according to a known permittivity distribution. For iterative image reconstruction algorithms, it is necessary to obtain the capacitance vector and compare it to the measurement vector, so that the image can be modified to reduce the difference between the two vectors.

There are three ways to solve the forward problem in ECT:

(1) Measuring the inter-electrode capacitance with a known permittivity distribution on an experimental setup [9].

(2) Using linear forward projection (LFP) [10].

(3) Calculating the capacitance using a computer model and a FEM solver.

The first approach has several disadvantages because it is difficult to set up a known permittivity distribution, measurement uncertainties, limitations and the restriction to a certain number of electrodes and configurations of the sensor. The second approach is limited by the use of the linearized response of the sensor to calculate the capacitance vector. Therefore, the third option, consisting of the use of a FEM solver is the most flexible and adequate technique to solve the forward problem. The interelectrode capacitance can be obtained by [11]

$$
C=\frac{Q}{V}=-\frac{1}{V} \iint_{\Gamma} \varepsilon(x, y) \nabla \varphi(x, y) d \Gamma
$$

Where $\mathrm{C}$ is the inter-electrode capacitance, $\mathrm{Q}$ is the surface charge density in the detection electrode, $\varepsilon$ is the permittivity at coordinates $(x, y), \phi$ is the potential at coordinates $(x, y)$, and $\Gamma$ is the electrode surface.

\subsection{Database implementation and construction}




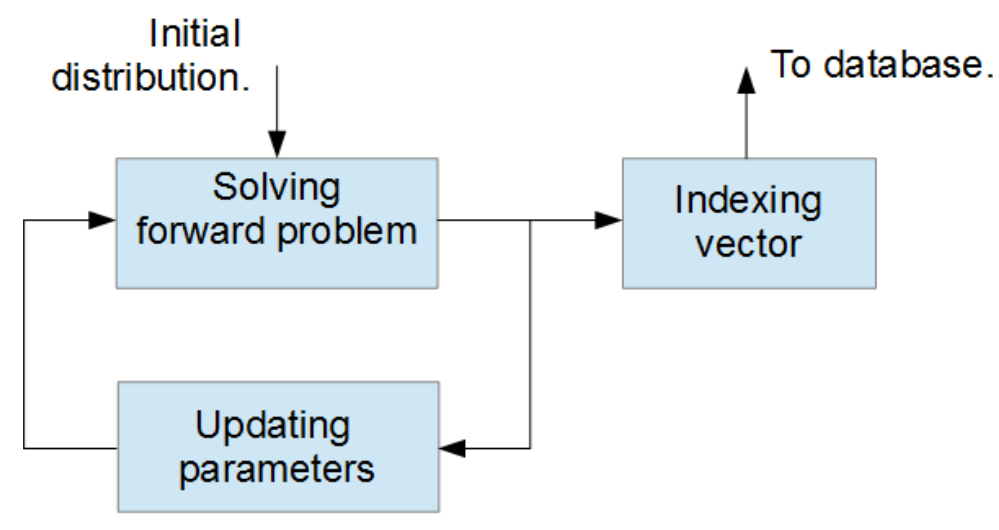

Figure 3 Database generation flowchart.

In the conventional model-based algorithm with an explicit model, an image is first reconstructed using a single-step algorithm, such as LBP, and then is used as a first attempt to estimate the parameters defining the model. Subsequently, the FEM solver computes the capacitance vector from to the first guess and the difference between the computed and measurement vectors is used to define how the parameters should be changed. Again, the FEM solver is used to compute the capacitance vector. This process is repeated until a desirable discrepancy between the computed and measured vectors is achieved. Using an explicit model, as shown in Figure 1, the fixed number of parameters can only be changed within a certain range. In consequence, the FEM solver is used repeatedly to compute the capacitance vector of a slightly changed permittivity distribution iteration after iteration. Furthermore, once a frame is successfully processed and the minimum is achieved, the process is repeated again for the next frame. In this iterative process, the same FEM model is solved repeatedly for each frame as the capacitance vectors computed are not stored. This represents a waste of computing power and time. In consequence, the conventional model-based algorithm is limited for off-line parameter estimation.

In the proposed approach, the database, containing a set of prior solved forward problems allows eliminating the use of the FEM solver for computing the forward problem on each iteration. Such a database is generated in advance by computing just once and storing the capacitance vector for a group of possible configurations of the parameters defining the model.

The characteristics of the 2D FEM model used to calculate the database are:

- Sensor diameter: $10 \mathrm{~cm}$

- Number of electrodes: 8

- Mesh elements: 724

To guarantee the accuracy of the FEM simulation, a comparison between the normalised capacitance obtained with a normal mesh formed by 724 elements and a finer mesh with 2040 elements, as shown 
in Figure 4, is performed. As shown in Figure 5, the difference between the two results is less than 2\%, confirming the consistency of the results of meshes with different mesh density.

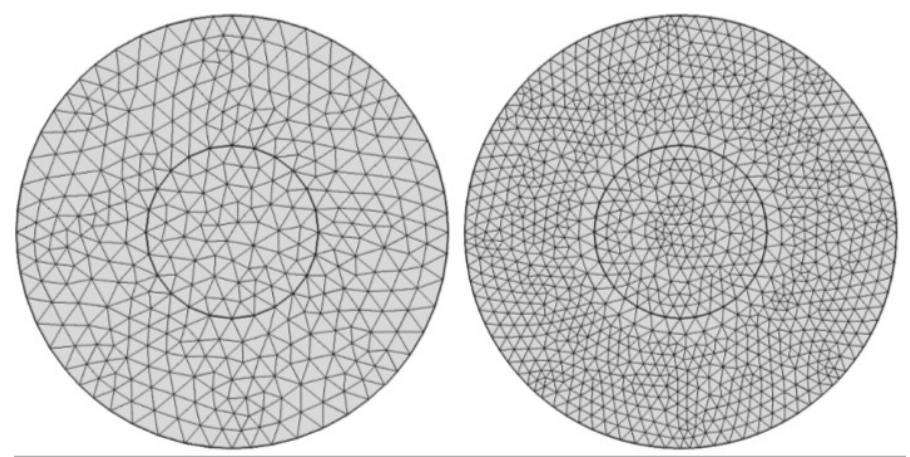

Figure 4 FEM mesh. Normal (left), finer (right).

The dimension of the database depends on the number of parameters selected to define the model. Whereas its size depends on the range and steps for the variation in the parameters. Figure 6 shows a $3 \mathrm{D}$ arrangement for a two-parameter model. If the parameters in Figure 1 (b) are selected to define the model, with an 8-electrode sensor; the size of the arrangement in the x-direction would be 28 according to

$$
M=\frac{n(n-1)}{2}
$$

where $\mathrm{M}$ is the number of independent measurements and $\mathrm{n}$ is the number of electrodes.

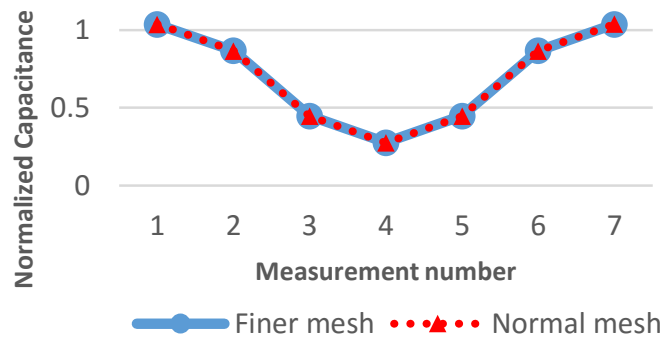

Figure 5 Normalised capacitance for first 7 measurements. Circle, finer mesh with 2040 elements. Triangle, normal mesh 724 elements. 


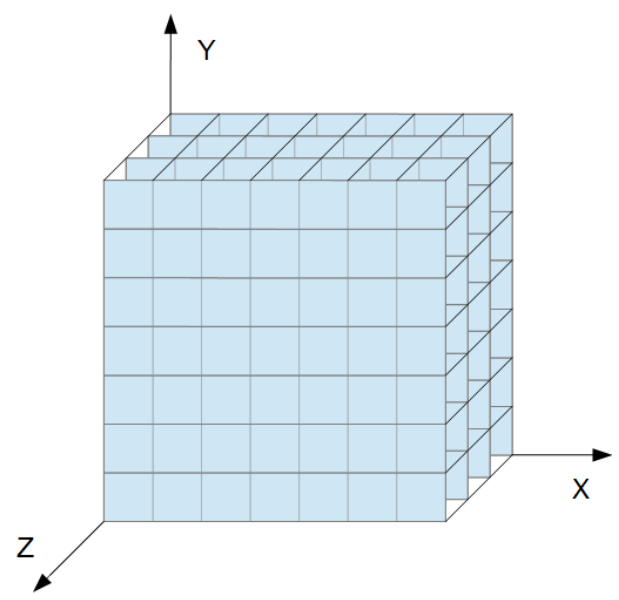

Figure 63 database. Xaxis measurement vector. $Y$ axis radius variation, Zaxis permitivity variation.

\subsection{Error calculation}

The measurement vector is compared to the vectors in the database to find the one that minimises the error. The capacitance vector is composed of an M number of elements as defined by equation 3 . The error is calculated using the norms of the vectors.

$$
\text { error }=\frac{\left\|C_{d b}-C_{m}\right\|}{\left\|C_{d b}\right\|}
$$

where $\mathrm{C}_{\mathrm{db}}$ is each normalised vector contained in the database, $\mathrm{C}_{\mathrm{m}}$ is the normalised measurement vector.

\section{Test case: Multi-component gas/oil/water flow}

In oil extraction, water and gas are produced alongside oil. It is usual to find water alongside oil and gas as a form of brine with dissolved solids. Water flooding is also common in oil extraction, i.e. injecting water into the reservoir to increase the pressure and maximise the production. The water constituting part of the liquid phase can be present as "free" water, i.e. not entrapped in the oil. It is also possible to find the water as an emulsion. Depending on the amount of water being produced, the liquid phase can be considered oil-continuous or water-continuous. Although the inversion point is not precisely determined, it is considered that a water-oil mixture with a content of more than $50 \%$ oil is oil-continuous, and more than $50 \%$ of water to be water-continuous [12]. In addition, $100 \%$ oil or water cannot be discarded as a possible composition of the liquid phase. Water cut, also known as water-inliquid ratio (WLR) is used as an indicator of the amount of water present in the liquid phase. The water volume fraction of the liquid phase can be defined by

$$
W L R=\frac{\text { Water volumetric flow rate }}{\text { Total liquid volumetric flow rate }}
$$


The relationship between the permittivity and conductivity of liquid mixture and the WLR for an oilcontinuous and water-continuous liquid phase is expressed by [13]

$$
\begin{gathered}
\varepsilon_{\text {liq }}^{\text {oil }}=\varepsilon_{\text {oil }} \frac{1+2 W L R}{1-W L R} \quad \sigma_{\text {liq }}^{\text {oil }}=\sigma_{\text {oil }} \frac{1+2 W L R}{1-W L R} \\
\varepsilon_{\text {liq }}^{\text {water }}=\varepsilon_{\text {water }} \frac{2 W L R}{3-W L R} \quad \sigma_{\text {liq }}^{\text {water }}=\sigma_{\text {water }} \frac{2 W L R}{3-W L R}
\end{gathered}
$$

where $\varepsilon_{\text {liq }}^{\text {oil }}$ is the permittivity of the oil-continuous liquid phase, $\varepsilon_{\text {oil }}$ is the permittivity of oil, $\varepsilon_{\text {liq }}^{\text {water }}$ is the permittivity of the water-continuous liquid phase, $\varepsilon_{\text {water }}$ is the permittivity of water, $\sigma_{\text {liq }}^{\text {oil }}$ is the conductivity of oil-continuous liquid mixture, $\sigma_{\text {oil }}$ is the conductivity of oil, $\sigma_{\text {liq }}^{\text {water }}$ is the conductivity of the water-continuous liquid mixture and $\sigma_{\text {water }}$ is the conductivity of water.

ECT systems have a limit on the amount of water present in the liquid mixture. This limit has been proven to be $30 \%$ [14].

Natural gas is usually present in reservoirs. Alongside with water, it generates pressure over the oil to push it up the well in the first stages of oil extraction. Once the pressure is decreased because oil left the reservoir, gas is injected to increase the pressure. Usually, natural gas is reinjected into the well. Air and carbon dioxide are also commonly used [15]. The increasing amount of gas inside the reservoir is reflected in the amount of gas being transported upwards throughout the pipe. Void fraction or gas void fraction (GVF) is used as an indicator of the amount of gas in relationship to the total volume [16].

$$
G V F=\frac{\text { Gas volumetric flow rate }}{\text { Total volumetric flow rate }}
$$

\subsection{Flow conditioner}

The use of a flow conditioner allows constraining the flow pattern so that the real flow can be described accurately by a selected model. In the case of gas/oil/water flow, a Venturi tube is used to accelerate the induced swirl in the flow. Due to the higher density of the oil/water mixture, the centrifugal force can push the liquid mixture towards the pipe wall, generating an annular distribution as seen in Figure 7 (a). The external liquid layer consists of an emulsion of oil/water. Whereas the central region is composed of the less dense gas core [14].

Another possible configuration for this annular flow pattern is an off-centre gas core, as shown in Figure 7 (b) because the swirling induced in the flow can also generate the displacement of the gas core away 
from the centre. In this case, a new set of parameters is required to define the flow pattern, indicating the location of the centroid of the gas core, i.e. the $\mathbf{x}$ and $\mathbf{y}$ coordinates for the centre of the gas core.

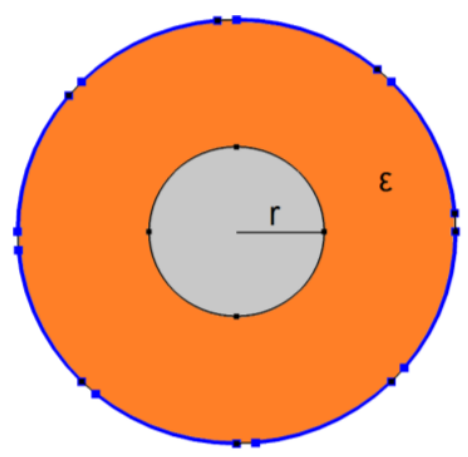

(a) Annular central core flow distribution, Gas core radius (r) and liquid permittivity $(\varepsilon)$.

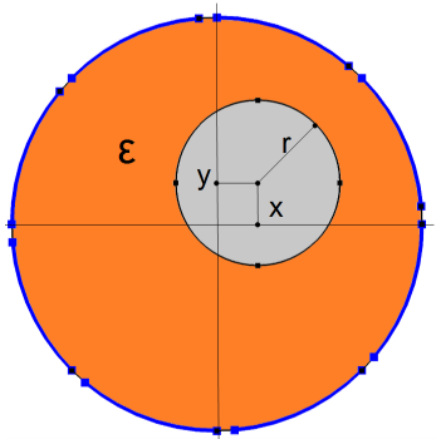

(b) Annular off-centre core flow distribution, Liquid mixture permittivity $(\varepsilon)$, gas core radius $(r)$ and centroid coordinates $(\mathrm{x}, \mathrm{y})$

Figure 7 parameters of annular flow distribution

As the distribution shown in Figure 7 (a) is a special case of the distribution depicted in Figure 7 (b) when the $\mathbf{x}$ and $\mathbf{y}$ parameters are zero, only one database is required for characterising the annular flow pattern for both, centred and off-centre gas core. The resultant database consists of a 5D array of size $30,28,12,12,9$ respectively on each dimension. The first dimension of the database is defined by the steps on the radius increment. The second dimension is due to the number of independent measurements. The third and fourth dimensions are defined by the number of steps on the $\mathbf{x}$ and $\mathbf{y}$ position respectively. Finally, the fifth dimension is defined by the number of steps in the permittivity change. Table 1 summarises the range and step length for parameter variation.

Table 1 Ranges and step length for parameter variations in database construction.

\begin{tabular}{|c|c|c|c|}
\hline Parameter & Range & Step size & Step size \% of full range \\
\hline Radius $\mathbf{r}$ & $.1-3 \mathrm{~cm}$ & $.1 \mathrm{~cm}$ & $3.5 \%$ \\
\hline Position $\mathbf{x}$ & $0-1.2 \mathrm{~cm}$ & $.1 \mathrm{~cm}$ & $9 \%$ \\
\hline Position $\mathbf{y}$ & $0-1.2 \mathrm{~cm}$ & $.1 \mathrm{~cm}$ & $9 \%$ \\
\hline Permittivity $\boldsymbol{\varepsilon}$ & $2-6$ & .5 & $12 \%$ \\
\hline
\end{tabular}

\subsection{Parameter calculation}

The parameters $\mathbf{r}, \mathbf{x}, \mathbf{y}, \boldsymbol{\varepsilon}$ are found by locating a vector in the database that minimises the error. Calculated by equation 4 , the vector that minimises the error is related to the parameters by the indexes of the database. 
In the case of the off-centre gas core, the area inside the sensor is divided into four quadrants, as shown in Figure 8. Variation in parameters $\mathbf{x}, \mathbf{y}$ in the generation of the database is from the centre of the sensor into the quadrant 1 . Variation of the parameters is not performed for quadrants 2, 3, 4 .

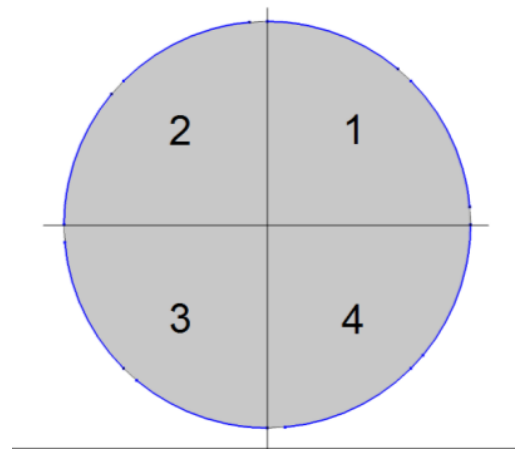

Figure 8 four quadrants

With the circular symmetry of an ECT sensor, it is not necessary to perform the time-consuming calculation of the database for the remaining quadrants. The measurement vector is rotated to match the vectors contained in the database as shown in Figure 9.

ORIGINAL VECTOR

1-2 1-3 1-4 1 -5 $1-6$ 1-7 $1-8$ 2-3 2-4 2-5 2-6 2 2-7 2-8 3-4 3-5 3-6 3-7 $3-8$ 4-5 4-6 4-7 4-8 5-6 5-7 5-8 6-7 6-8 7-8

1ST ROTATION

3-4 3-5 3-6 3-7 3-8 1-3 2-3 4-5 4-6 4-7 4-8 1-4 2-4 5-6 5-7 5-8 1 1-5 $2-5$ 6-7 6 6-8 1 1-6 $2-6$ 7-8 1-7 2-7 1-8 2-8 1 -2

2ND ROTATION

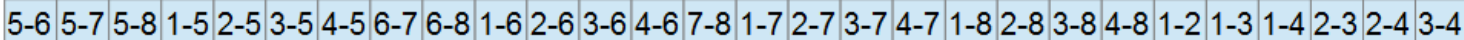
3RD ROTATION

7-8 1-7 2-7 3-7 4-7 5-7 6-7 1-8 2-8 3-8 4-8 5-8 6-8 1-2 1-3 1-4 1-5 1-6 2-3 2-4 2-5 2-6 3-4 3-5 3-6 4-5 4-6 5-6

Figure 9 Vector rotation. Electrode pair measurements.

Figure 10 shows the effect of rotating the measurement vector to displace the centre of the gas core from 2, 3 and 4 quadrants to the quadrant 1 . In this way, it is possible to find the four distinctive parameters for the gas core on any quadrant of the sensor.

Due to the size of the 5D database containing the measurement vectors, parameter calculation on CPU is time-consuming. To reach the minimum error in an adequate time and to perform real-time monitoring, the proposed algorithm has been implemented in a parallel computing hardware. The use of MATLAB ${ }^{\circledR}$ language alongside with the CUDA ${ }^{\circledR}$ platform allows the fast implementation of parallel processing routines on NVIDIA ${ }^{\circledR}$ general-purpose graphics processing unit (GPU). A major advantage of using CPU and GPU is the ability to run the same algorithm without changes on both, GPU and CPU hardware. The calculation is performed on a Dell laptop with a dual core i7 processor 
running at $2.8 \mathrm{GHz}$. GPU calculation is performed on the same computer. The on-board GPU is a NVIDIA ${ }^{\circledR}$ GeForce GT 750m.

Rotation

Original vector

$1^{\text {st }}$ rotation

$2^{\text {nd }}$ rotation

$3^{\text {rd }}$ rotation
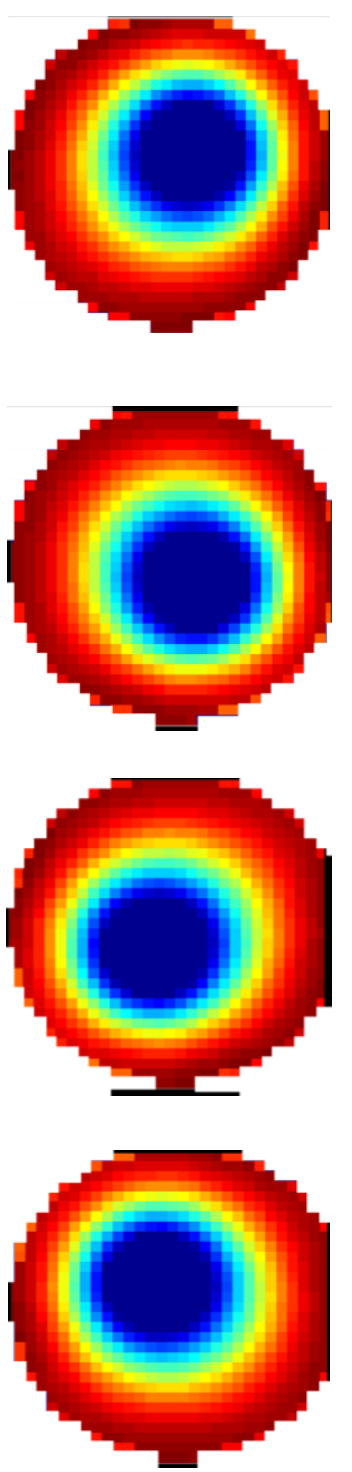

Capacitance vector

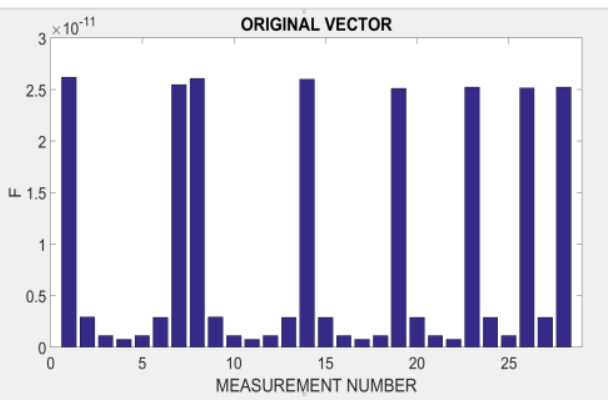

FIRST ROTATION

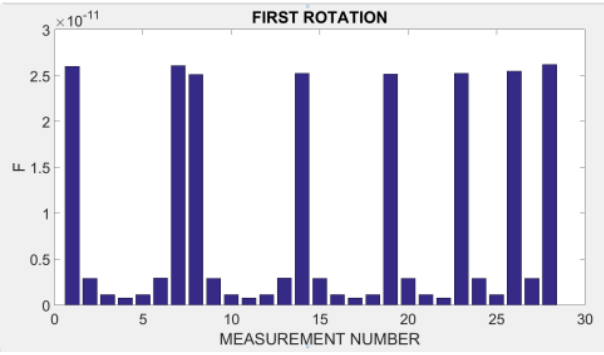

SECOND ROTATION
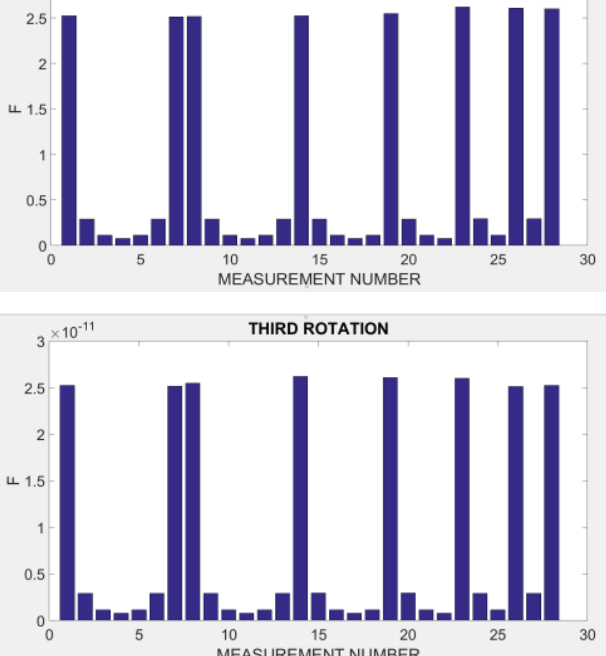

Figure 10 Image reconstruction after vector rotation

To test the time required to compute the parameters using both architectures, a sample of 500 noisefree capacitance vectors is used to perform image reconstruction. Figure 11 shows the time required for each iteration in both architectures. Computation on GPU takes an average of $7.4 \mathrm{mS}$. Whereas computation on CPU takes an average of $91.1 \mathrm{mS}$, it is possible to see from this results that calculation of GPU is around 12 times faster. In consequence, the implementation of the proposed algorithm in parallel computing hardware allows performing real-time computation for ECT systems with a frame rate up to 100 frames/second. 


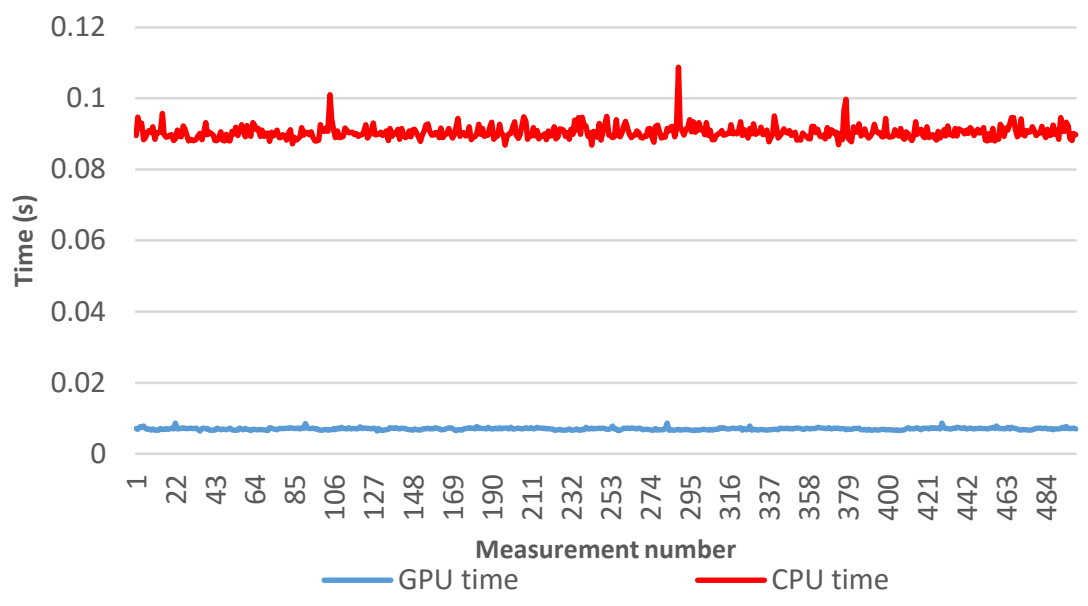

Figure 11 GPU versus CPU time performance.

\section{Simulation Results}

Simulation was performed to evaluate the model-based algorithm with databases. A comparison between the proposed approach and LBP algorithm is presented. The model-based algorithm without databases is not included in the performance comparison because such an algorithm cannot be used for real-time monitoring due to its time-consuming nature. LBP images area characterised for showing smooth transitions on the boundaries between materials; this complicates the task of measuring the parameters defining the model. In consequence, the image needs to be binarized to allow the calculation of the radius of the gas core. Ideally, pixels in the LBP images have values between 1 and 0 . Although this is not always the case, especially when the permittivity of the materials does not reach the high calibration point. Therefore, a medium point between the value of the pixel with the highest and lowest value needs to be set as a threshold. Because of this, pixels with values above the average value are set to 1 . In contrast, pixels with values below the average are set to 0 . Once the image is binarized, it is possible to apply the regionprops MATLAB built-in function to calculate the centroid and radius of the central gas core.

For all simulation tests, four sets of data are used to perform the comparison: (1) a set of noise-free data, (2) $100 \mathrm{~dB}$ SNR data, (3) $50 \mathrm{~dB}$ SNR data and (4) $30 \mathrm{~dB}$ SNR data. Making use of the AWGN built-in MATLAB function, white Gaussian noise is added to the capacitance vector before normalisation. The SNR is given by the ratio between the power of the signal and the noise as defined by

$$
S N R_{d B}=20 \log _{10}\left(\frac{A \text { signal }}{\text { A noise }}\right)
$$

Where $S N R_{d B}$ is the signal to noise ratio in decibels, $A$ signal is the amplitude of the signal and $A$ noise is the amplitude of the noise. 
The first test consists of the calculation of the radius with a fixed permittivity. The radius varies from 0.1 to $4.9 \mathrm{~cm}$ whereas the permittivity is set to 6 . Figure 12 shows the error for radius calculation with LBP and model-based algorithms. The capacitance vectors are normalised with low and high permittivity calibration values set at 1 and 15 respectively.

Excellent agreement can be seen from Figure 12 (b) between the real value and the value calculated with the model-based algorithm for the estimation of the radius of the gas core with low noise levels. However, the estimation from the LBP algorithm diverges greatly from the real value as seen from Figure 12 (a), especially for small radius because of the characteristic low spatial resolution of ECT. As the centre of the gas core was placed at the centre of the sensor, it is well known that the sensitivity in ECT systems decreases sharply towards the centre of the sensor. This generates a further error when the radius is calculated with images obtained with LBP. The low sensitivity on the centre of the sensor also affects the response of the model-based algorithm for data with a low SNR. Although the response of the model-based algorithm is much more sensitive to higher noise levels than the LBP algorithm, the absolute error remains lower than the error obtained for the LBP algorithm.

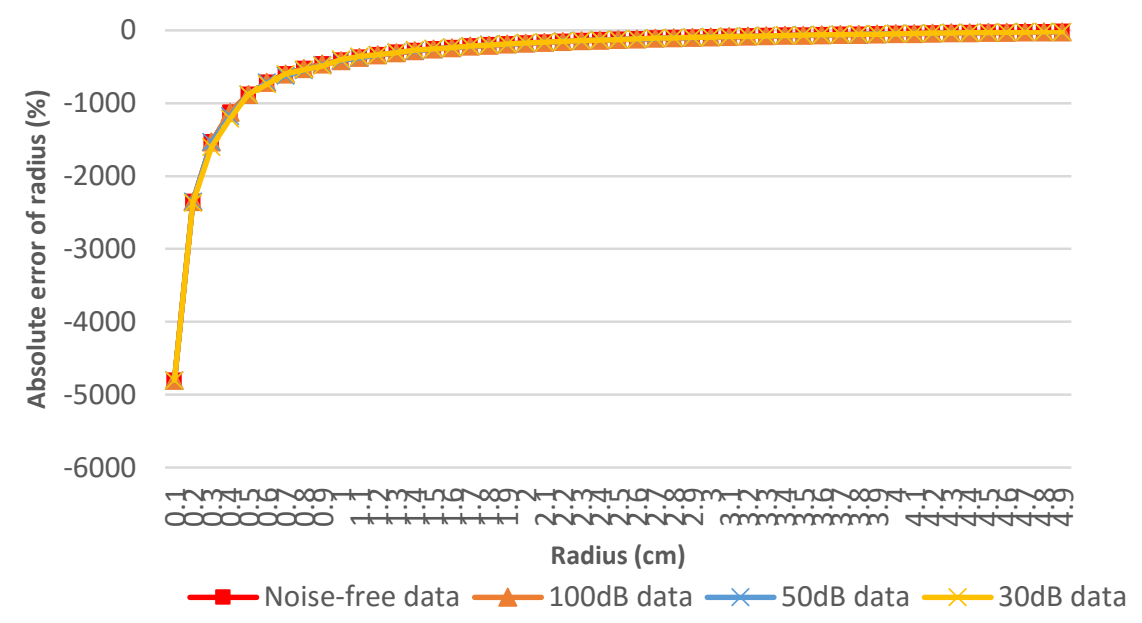

(a) LBP radius calculation error. 


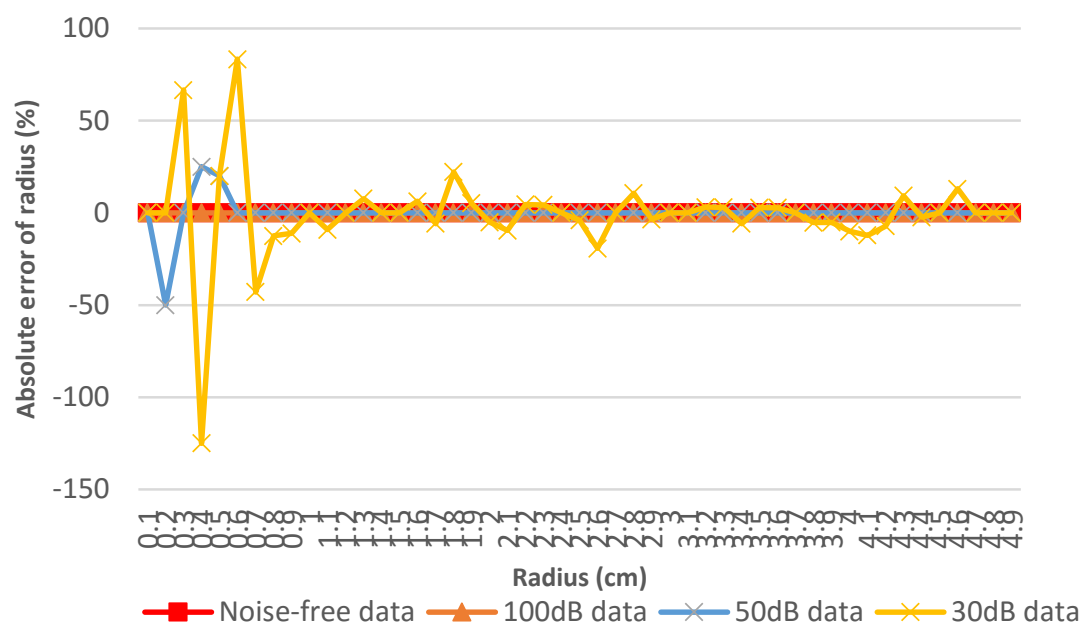

(b) Model-based radius calculation error.

Figure 12 Radius calculation error with different approaches (a) LBP (b) Model-based.

For the second test, the radius of the gas core is fixed. In this case, at $3 \mathrm{~cm}$ whereas the permittivity of the liquid is changed from 1.5 to 15 . As with the previous test, the capacitance data is normalised with the low and high calibration points set to 1 and 15 respectively. Figure 13 shows the error for permittivity calculation with LBP and model-based algorithms respectively. It can be seen from Figure 13 (a) that although the LBP algorithm performs better for estimating the permittivity than the radius, there still exists a considerable deviation from the real value. Furthermore, for higher permittivity values the error increases. In contrast, the permittivity estimation from the model-based algorithm shown in Figure 13 (b) shows an excellent agreement with the real value, even for data with high noise levels. Results shown in Figure 13 are expected because the liquid ring is near to the pipe wall. In this area, the sensitivity is greater and measurements are less sensitive to high noise levels.

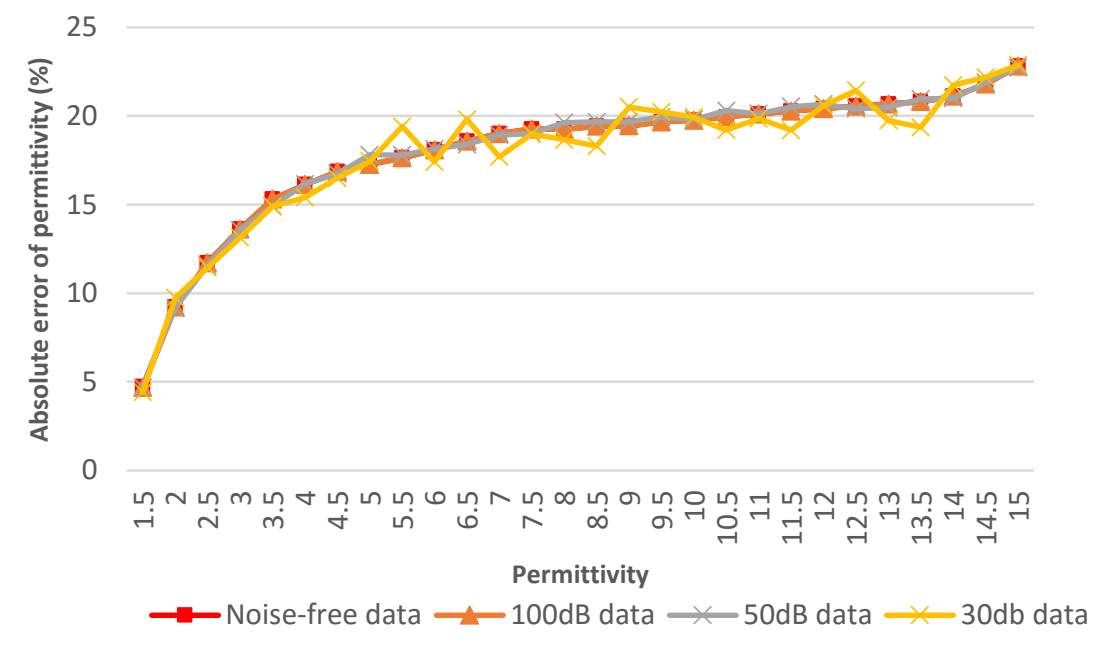

(a) LBP permittivity calculation error. 


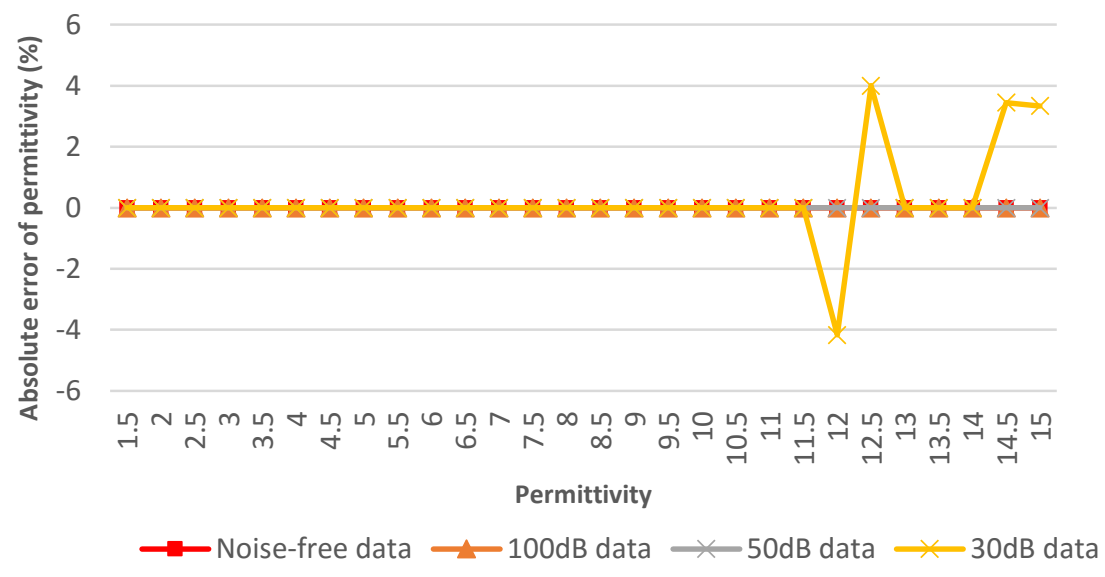

(b) Model-based permittivity calculation error.

Figure 13 Permittivity calculation error with different algorithms. (a) LBP (b) Model-based.

Figure 14 shows simulation results for radius calculation for an off-centre gas core. For this test, the permittivity of the liquid mixture is set to 4 and the centroid of the gas core is set to 1.2 in both $X$ and $Y$ coordinates according to the model described in figure 7 (b).

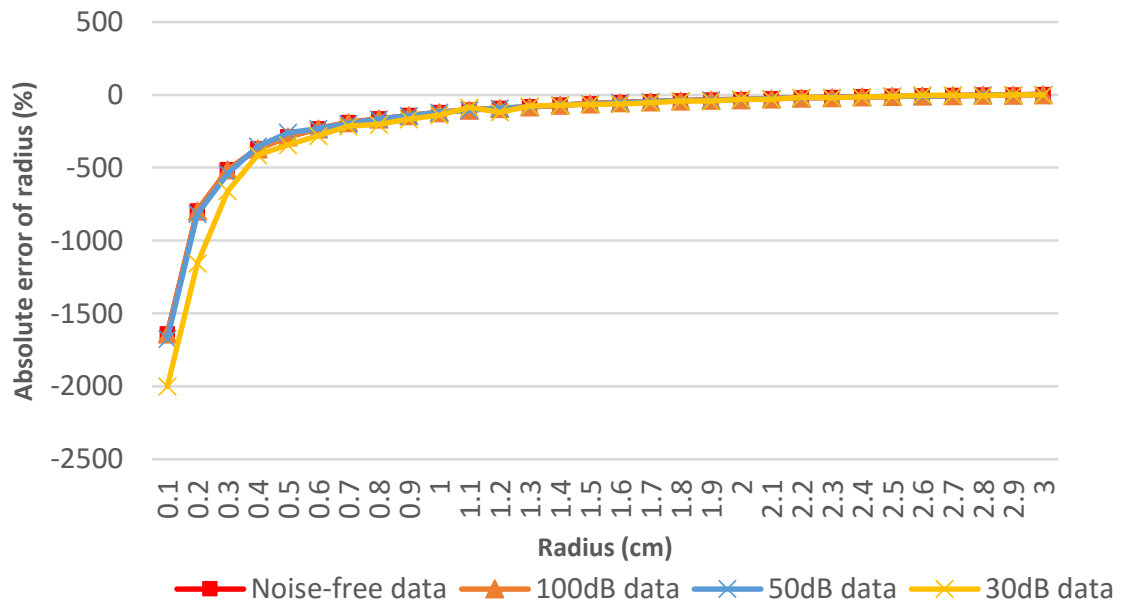

(a) LBP radius calculation error. 


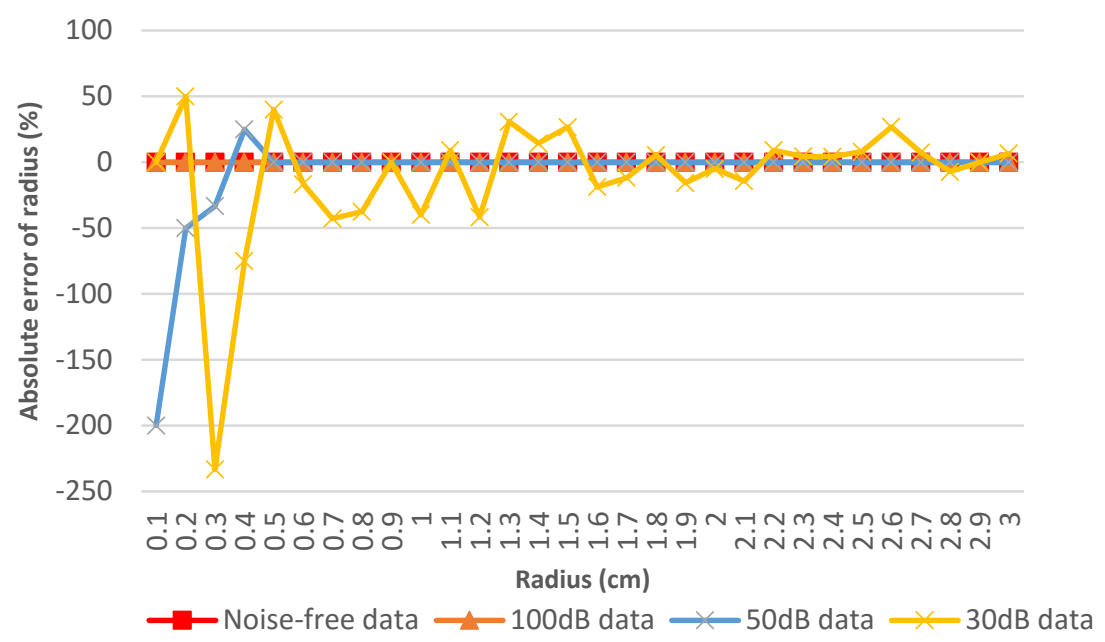

(b) Model-based radius calculation error.

Figure 14 Radius calculation error for the off-centre gas core with different algorithms (a) LBP (b) Model-based.

Figure 15 shows the simulations results for the determination of the $\mathrm{X}$ coordinate for a model as described in figure 7 (b). During this test, the permittivity of the liquid mixture is kept constant at 4, the radius at $2.5 \mathrm{~cm}$ and the $\mathrm{Y}$ coordinate set at $0 \mathrm{~cm}$.

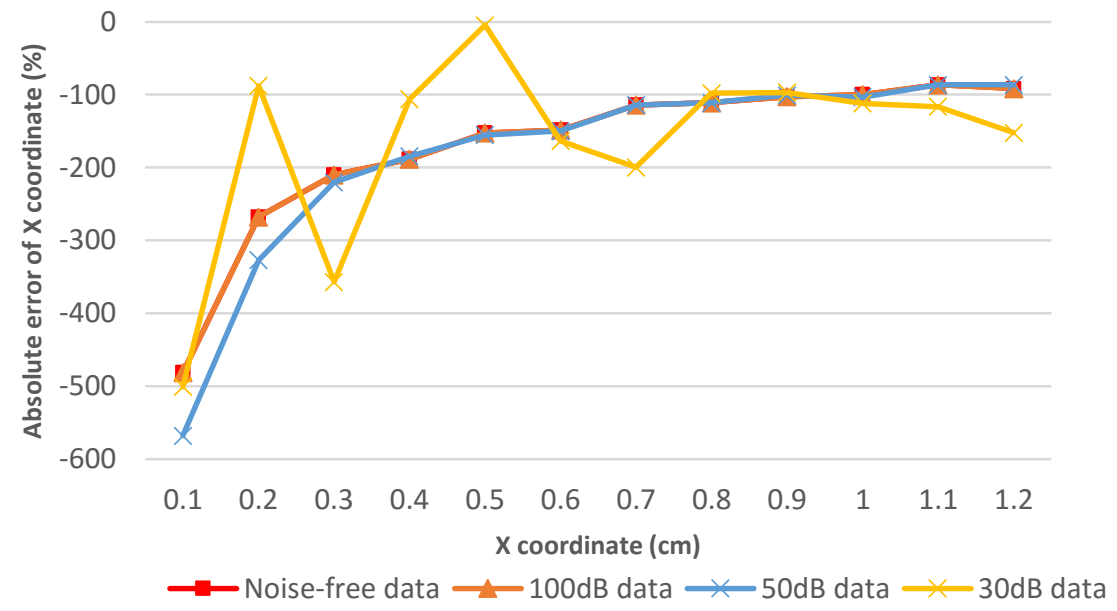

(a) LBP coordinate calculation error. 


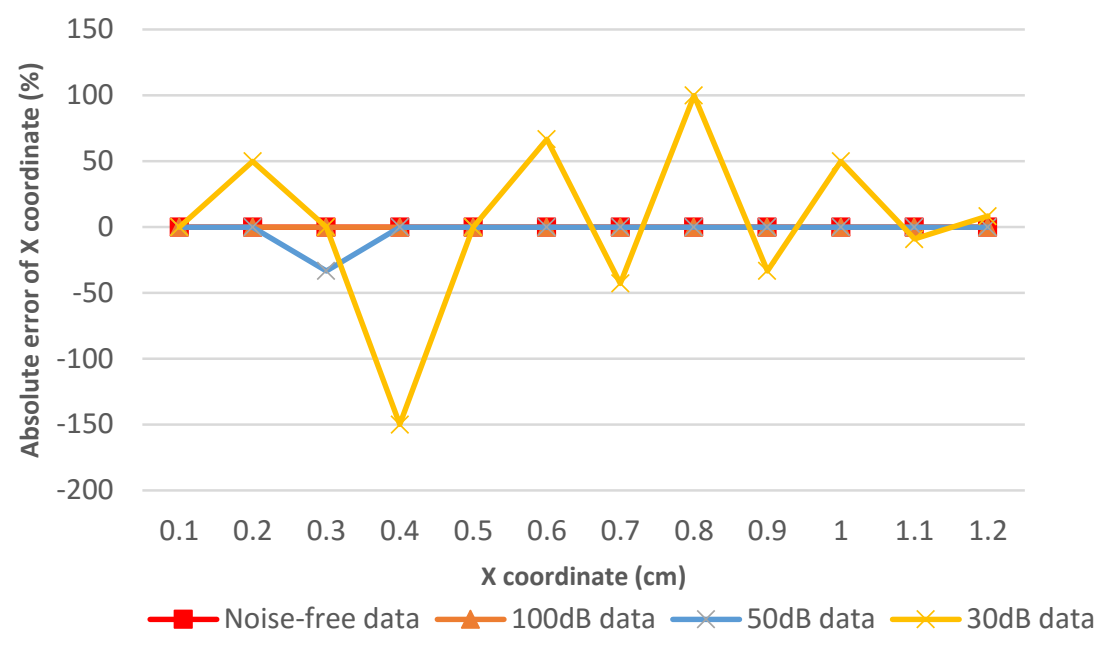

(b) Model-based coordinate calculation error.

Figure $15 \mathrm{X}$ coordinate calculation error with different algorithms. (a) LBP (b) Model-based.

Figure 16 shows the simulation result for the estimation of the parameter $\mathbf{d}$ according to the stratified flow model depicted in figure 1 (a). For this test, the permittivity of the liquid mixture is set to 4.8 and the angle $\boldsymbol{\theta}$ is set to $-90 \mathrm{deg}$. It is possible to notice from figure 16 (a) the high error levels throughout the full range of the parameter $\mathbf{d}$. Due to the smooth shape of the sensitivity distribution in the sensing area, it is not possible to achieve sharp boundaries when reconstructing the image with the LBP algorithm. In consequence, the estimation of the parameter $\mathbf{d}$ generates great deviations from the real values.

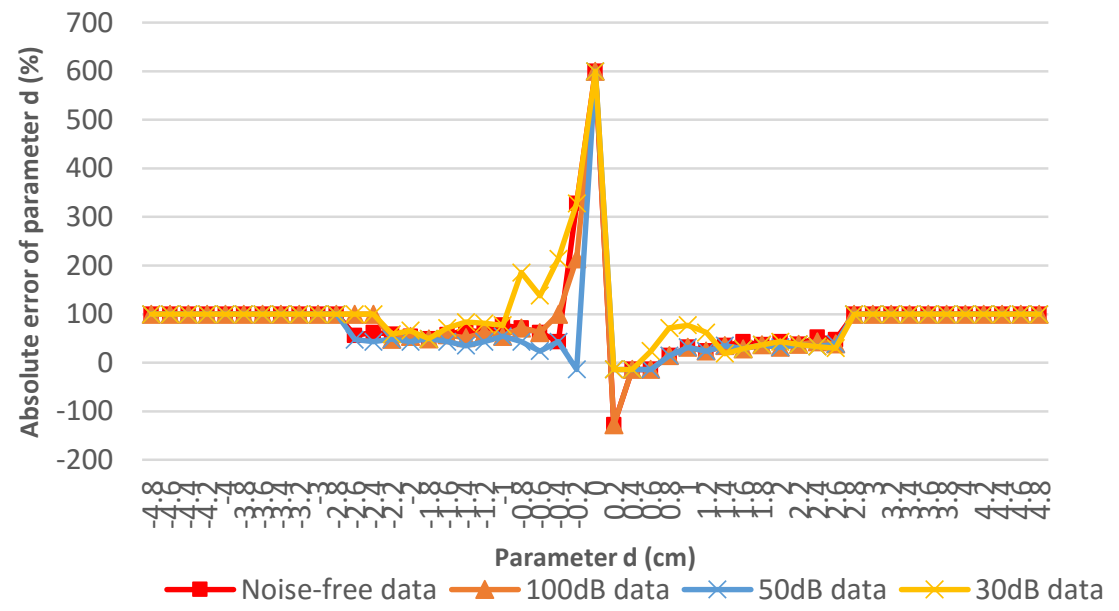

(a) LBP parameter d calculation error. 


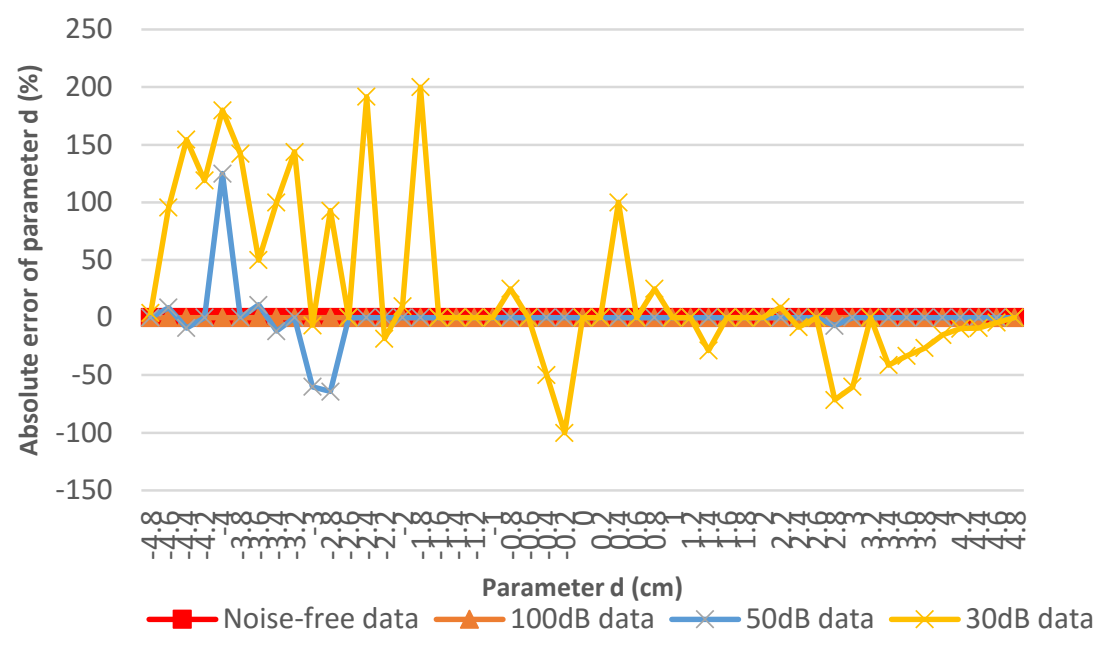

(b) Model-based parameter d calculation error.

Figure 16 Parameter d calculation error with different algorithms (a) LBP (b) Model-based.

Figure 17 shows the estimation of the permittivity from simulated data. For this test, the parameter $\mathbf{d}$ is set to $0 \mathrm{~cm}$. The angle $\boldsymbol{\theta}$ is set to $-90 \mathrm{deg}$. As seen on the results from figure 13 , the proximity of the liquid mixture to the high sensitivity area near the pipe wall allows the estimation of the permittivity of the liquid mixture with lower error levels and less noise sensitivity with both algorithms.

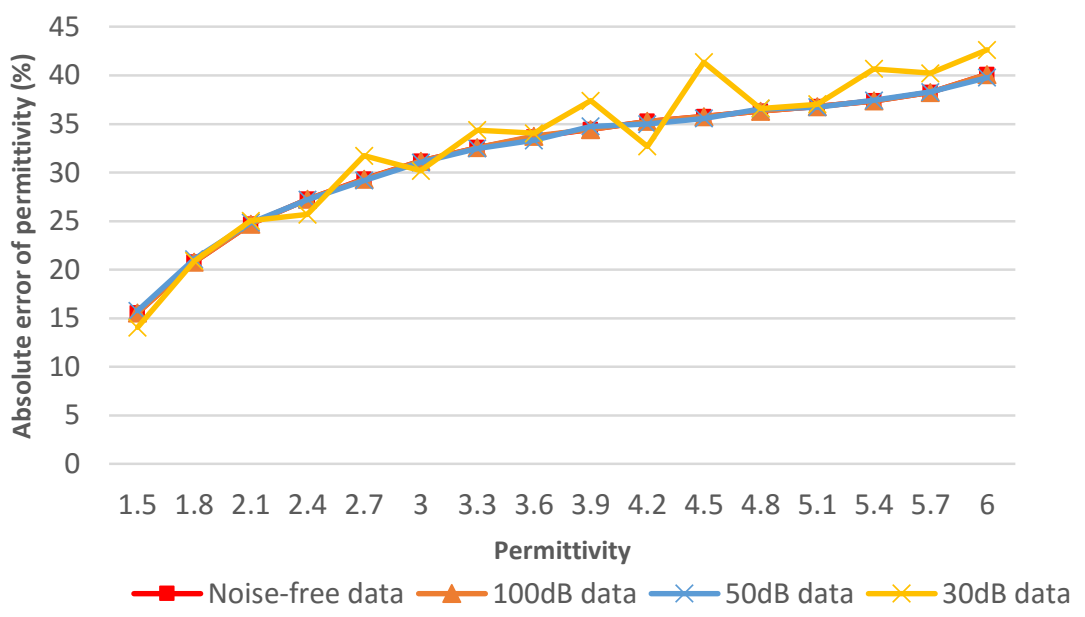

(a) LBP permittivity calculation error. 


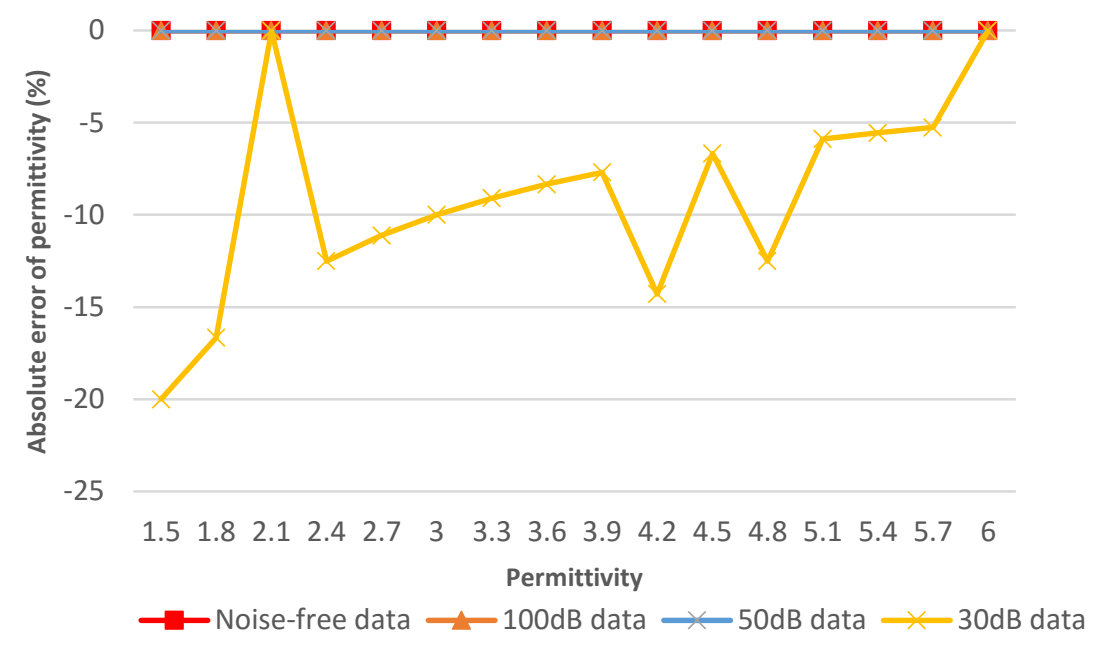

(b) Model-based permittivity calculation error.

Figure 17 Permittivity calculation error with different algorithms. (a) LBP (b) Model-based.

\section{Experimental validation}

To validate the proposed algorithm experimentally an impedance analyser-based ECT system was used, as shown in Figure 18. The excitation frequency was set to $500 \mathrm{kHz}$ and the voltage at $1 \mathrm{~V}$. The signalto-noise ratio of the system is $45 \mathrm{~dB}$ as calculated with equation 9 .

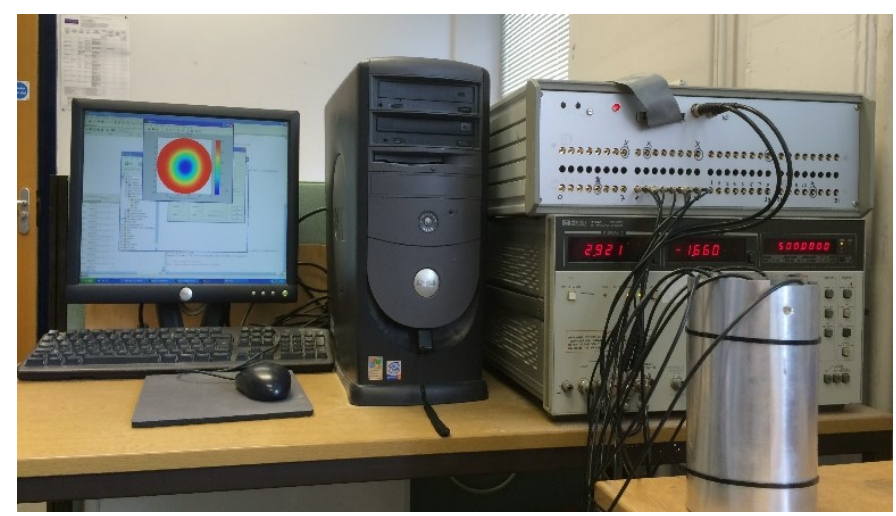

Figure 18 Impedance analyser based ECT system.

A permittivity distribution is set with an empty glass cylinder $(\mathrm{r}=1.85 \mathrm{~cm})$, representing the gas core $(\varepsilon=1)$ and an emulsion generated with tap water $(\varepsilon=80)$ and corn oil $(\varepsilon=1.6)$, as shown in Figure 19. The characteristics of the sensor with internal electrodes are the following:

- Number of electrodes $\mathrm{N}=8$

- Electrode width: $.5 \mathrm{~cm}$

- Inter-electrode radial gap: $1 \mathrm{~cm}$

- Container diameter: $7.3 \mathrm{~cm}$ 
- Wall thickness: $0.1 \mathrm{~cm}$

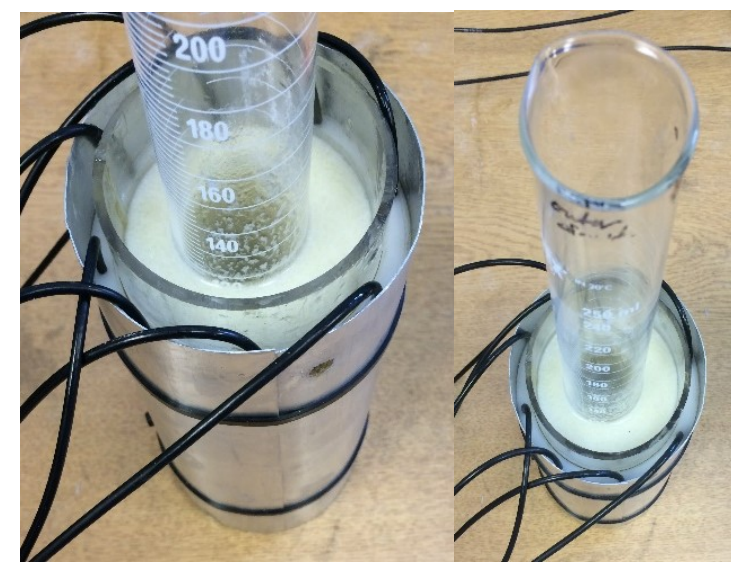

Figure 19 Real permittivity distribution. Water-oil emulsion ring. An empty tube as the gas core.

Three different measurements were obtained by using water/oil emulsions with different rates: (1) $0 \%$ WLR (100\% oil, $0 \%$ water), (2) 20\% WLR (80\% oil, $20 \%$ water) and (3) $30 \%$ WLR ( $70 \%$ oil, $30 \%$ water). The capacitance vector is normalised with the low and high permittivity values, set at 1 , sensor full of air, and 3.6, sensor full of 30\% WLR emulsion, respectively. Figure 20 shows the images reconstructed with the LBP algorithm alongside the binarized images.

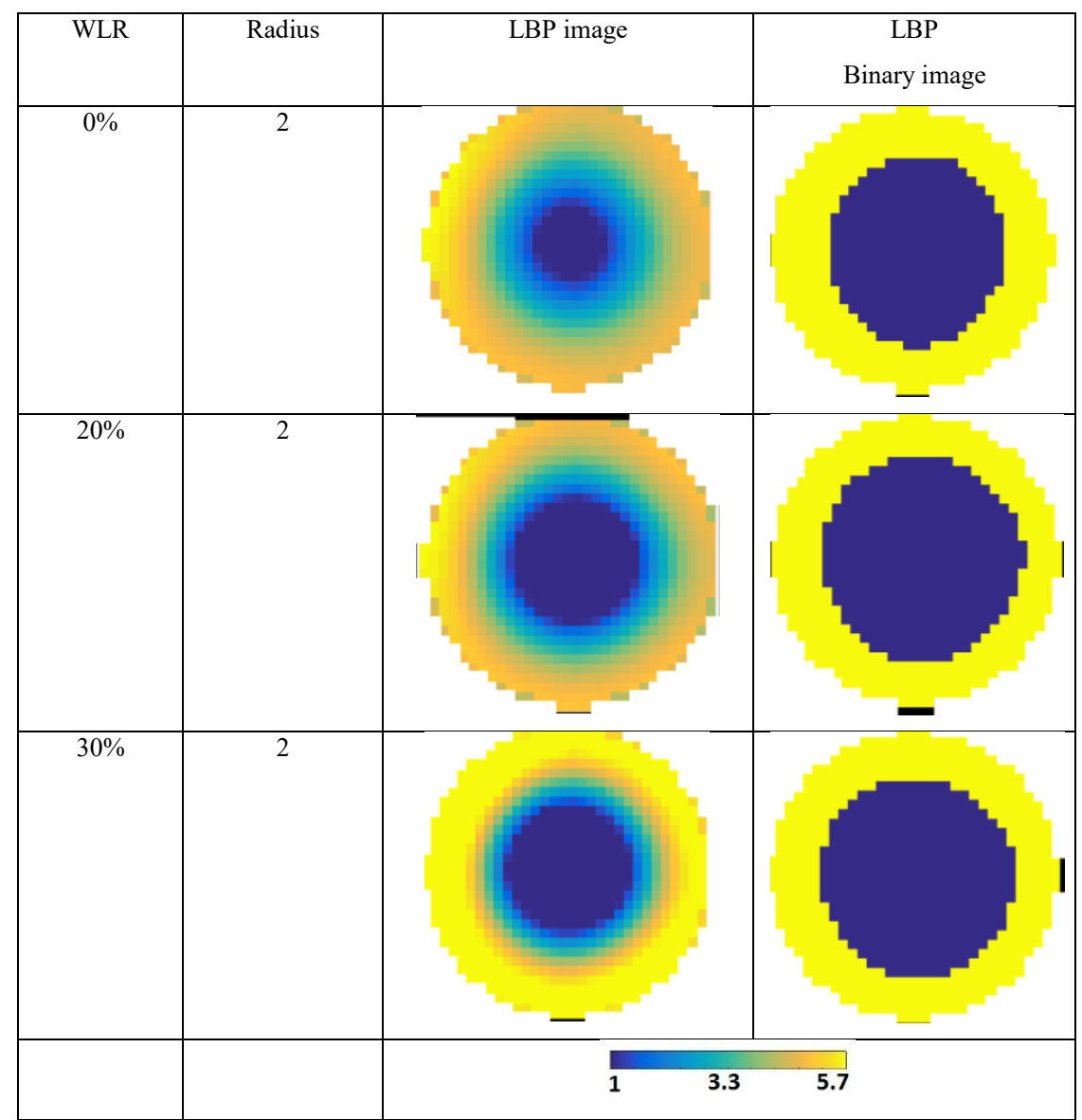

Figure 20 LBP and LBP binary images. 
Table 2 shows experimental results for the proposed approach. It is possible to notice from the results on table 2 and 3 that the low SNR achieved with the impedance analyser based ECT system affects the performance of the results. In addition, expanding the data points in the database would increase the accuracy of the measurements.

Table 2 Radius and permittivity estimation with LBP and model-based algorithm for a central gas core.

\begin{tabular}{|c|c|c|c|c|c|c|c|c|c|c|c|}
\hline \multirow{2}{*}{ GVF } & \multirow{2}{*}{ WLR } & \multirow{2}{*}{$\begin{array}{c}\text { Radius } \\
\text { (r) } \\
\mathrm{cm} / \mathrm{GVF}\end{array}$} & \multirow{2}{*}{$\begin{array}{c}\text { Theoretical } \\
\text { value } \\
(\varepsilon)\end{array}$} & \multicolumn{4}{|c|}{ LBP } & \multicolumn{4}{|c|}{ Model-based } \\
\hline & & & & $\mathrm{r}$ & $\varepsilon$ & GVF & WLR & $\mathrm{r}$ & $\varepsilon$ & GVF & WLR \\
\hline $24 \%$ & $0 \%$ & 1.8 & 1.6 & 1.6 & 1.3 & $21 \%$ & $-6 \%$ & 2.0 & 2 & $30 \%$ & $7 \%$ \\
\hline $24 \%$ & $20 \%$ & 1.8 & 2.8 & 2.0 & 3.1 & $30 \%$ & $23 \%$ & 1.8 & 2.5 & $24 \%$ & $15 \%$ \\
\hline $24 \%$ & $30 \%$ & 1.8 & 3.6 & 2.0 & 3.9 & $30 \%$ & $32 \%$ & 1.9 & 3.5 & $27 \%$ & $28 \%$ \\
\hline
\end{tabular}

Table 3 Radius and permittivity estimation with LBP and model-based algorithm for an off-centre gas core.

\begin{tabular}{|c|c|c|c|c|c|c|c|c|c|c|c|}
\hline \multicolumn{4}{|c|}{ Real Values } & \multicolumn{4}{c|}{ LBP } & \multicolumn{4}{c|}{ Model-based } \\
\hline GVF & WLR & X & Y & X & Y & GVF & WLR & X & Y & GVF & WLR \\
\hline $24 \%$ & $0 \%$ & 0.6 & 0.0 & 0.7 & 0.1 & $21 \%$ & $-6 \%$ & 0.6 & 0.0 & $30 \%$ & $7 \%$ \\
\hline $24 \%$ & $20 \%$ & 0.6 & 0.0 & 0.5 & 0.0 & $30 \%$ & $23 \%$ & 0.7 & 0.0 & $24 \%$ & $15 \%$ \\
\hline $24 \%$ & $30 \%$ & 0.6 & 0.0 & 0.5 & 0.1 & $30 \%$ & $32 \%$ & 0.6 & 0.0 & $27 \%$ & $28 \%$ \\
\hline
\end{tabular}

\section{Conclusions}

The model-based algorithm can reduce the possible solutions to the underdetermined inverse problem. This is because the number of unknowns is reduced drastically to a few of parameters that describe the permittivity distribution. Although the conventional model-based algorithm is successful in calculating a quantitative estimation of the permittivity distribution, solving the forward problem on each iteration makes this algorithm not suitable for on-line parameter estimation. Single-step algorithms can generate a fast estimation of the distribution. However, such images are a quantitative representation only. Therefore, such algorithms cannot be used for quantitative monitoring of complex distributions, like gas/water/oil flow. A model-based algorithm with sets of prior calculated forward problems successfully overcomes the problem of accurately estimating permittivity distributions in real time. This is because the tedious and time-consuming task of solving the forward problem is performed in advance. In addition to drastically reducing image reconstruction time, the use of databases reduces the hardware requirements on the equipment performing image reconstruction, because the error calculated with equation 4 is considerably computationally less expensive than solving the forward problem with a FEM solver on each iteration. 
Although in this work the test case is focused on an annular gas/oil/water flow, the flexibility of using a computational model to solve the forward problem in advance allows creating databases for a variety of permittivity distributions, making this approach flexible to be implemented in a wide range of applications.

In addition, as discussed by Sun and Yang [17] and Rodriguez-Frias and Yang [18]. ERT systems with voltage excitation (ERTv) use an identical sensor structure and measurement scheme, i.e. applying an alternating voltage and measuring the resultant current in the detection electrode. Having the same sensor structure and excitation-detection approach, the numbers of independent measurements on ECT and ERT are the same, as defined by equation 3. In consequence, the results shown in this paper of implementing the model-based algorithm with databases can be replicated with an ERTv system.

\section{Acknowledgements}

Marco Antonio Rodriguez Frias thanks the Mexican Council for Technology and Science (CONACyT) for supporting his study at The University of Manchester in the United Kingdom.

\section{References}

[1] W. Q. Yang and P. Lihui, "Image reconstruction algorithms for electrical capacitance tomography," Meas. Sci. Technol., vol. 14, no. 1, p. R1, 2003.

[2] M. Soleimani and W. R. B. Lionheart, "Nonlinear image reconstruction for electrical capacitance tomography using experimental data,” Meas. Sci. Technol., vol. 16, no. 10, pp. 1987-1996, Oct. 2005.

[3] C. Ortiz-Aleman, R. Martin, J. C. Gamio, and A. Nicolas, "Application of Simulated Annealing and Genetic Algorithms to the Reconstruction of Electrical Permittivity Images in Capacitance Tomography," in 3rd World Congress on Industrial Process Tomography, Baniff, Canada, 2003, pp. 794-799.

[4] Y. Li and W. Q. Yang, "Updating Sensitivity Maps in Landweber Iteration for Electrical Capacitance Tomography," in 5th World Congress on Industrial Process Tomography, Bergen Norway, pp. 1116-1123.

[5] O. Isaksen and J. E. Nordtvedt, "A New Reconstruction algorithm for use with Capacitance-Based Process Tomography,” Meas. Sci. Technol., vol. 4, pp. 1464-1475, 1993.

[6] F. Klug and F. Mayinger, "Impedance based flow reconstruction - A novel flow composition measuring technique for multi-phase-flows," Nucl. Eng. Des., vol. 146, no. 1-3, pp. 35-42, 1994.

[7] M. A. Rodriguez Frias and W. Yang, "Model-based image reconstruction for electrical capacitance tomography with a prior calculated database," in 2016 IEEE International Conference on Imaging Systems and Techniques (IST), 2016, pp. 350-355.

[8] Q. Marashdeh, W. Warsito, L.-S. Fan, and F. L. Teixeira, "A multimodal tomography system based on ECT sensors," Sensors Journal, ..., vol. 7, no. 3, pp. 426-433, 2007.

[9] F. Klug and F. Mayinger, "Novel impedance measuring technique for flow composition in multi-phase- 
flows," in 29th ASME/ AIChE/ ANS National Heat Transfer Conference, 1993, pp. 1-8.

[10] W. Yang, D. M. Spink, T. a York, and H. McCann, “An image-reconstruction algorithm based on Landweber's iteration method for electrical-capacitance tomography,” Meas. Sci. Technol., vol. 10, no. 11, pp. 1065-1069, Nov. 1999.

[11] a. M. Olmos, J. A. Primicia, and J. L. F. Marron, "Influence of Shielding Arrangement on ECT Sensors," Sensors, vol. 6, no. 9, pp. 1118-1127, Sep. 2006.

[12] Y. Li, W. Yang, D. Tsamakis, Z. Wu, C. Lenn, C. Xie, S. Huang, and A. Cutler, "Model-based image reconstruction algorithm for measurement of multiphase distributions,” 2010 IEEE Int. Conf. Imaging Syst. Tech. IST 2010 - Proc., no. 1, pp. 96-99, 2010.

[13] A. J. Jaworski and T. Dyakowski, "Measurements of oil-water separation dynamics in primary separation systems using distributed capacitance sensors,” Flow Meas. Instrum., vol. 16, no. 2-3, pp. 113-127, 2005.

[14] Y. Li, W. Yang, Z. Wu, and D. Tsamakis, "Gas / oil / water Flow Measurement by Electrical capacitance," 2012, pp. 1-6.

[15] T. Ray and R. Sarker, "Multiobjective Evolutionary Approach to the Solution of Gas Lift Optimization Problems,” 2006 IEEE Congr. Evol. Comput., pp. 10951-10957, 2006.

[16] A. Fakhru'l-Razi, A. Pendashteh, L. C. Abdullah, D. R. A. Biak, S. S. Madaeni, and Z. Z. Abidin, "Review of technologies for oil and gas produced water treatment,” J. Hazard. Mater., vol. 170, no. 2-3, pp. 530$551,2009$.

[17] J. Sun and W. Yang, "A dual-modality electrical tomography sensor for measurement of gas-oil-water stratified flows," Measurement, vol. 66, pp. 150-160, Apr. 2015.

[18] M. A. Frias Rodriguez and W. Yang, "Electrical resistance tomography with voltage excitation," in IEEE Int. Instrumentation and Meas.Tech. Conf. I2MTC, 2016, no. 1, pp. 1-6. 